\title{
CFD Simulation Evaluation for Flow Distribution of the Closed Chamber
}

\author{
Hong Bin ${ }^{1, a}$, Wang Hongmei ${ }^{2}$ \\ ${ }^{1}$ Internal Combustion Engine Research Institute of Tianjin University, China \\ ${ }^{2}$ Tianjin Tianbo Science \& Technology Co,.Ltd., China
}

\begin{abstract}
Taking a closed chamber flow field as object, this paper establishes the 3D model of the Closed Chamber. With the conclusion of flow distribution and gasoline vapor concentration distribution of the Closed Chamber reached via the CFD software of Fluent and experiment research, this paper analyzes the flow distribution and concentration distribution of gasoline vapor. Several improvement projects are proposed, such as improving the wind circulation system to optimize the indoor gas concentration. By improving the structure of wind circulation system, it makes flow distribution more reasonable and significantly improves the uniformity of indoor gas concentration. Through implementation of this improvement, the reliability and performance of the containment chamber obviously program to improve.
\end{abstract}

\section{Submitting the manuscript}

With the development of computer technology, the use of computers to solve numerical simulation of indoor air control equations has also been greatly developed. This method is a combination product of computer technology and hydrodynamics, namely computational fluid dynamics CFD. Today, CFD simulation has been more and more into each field and gradually become one indispensable tool of the research projects.

With the rapid development of motorcycles manufacturing industry, pollution emissions of motorcycles have gradually been concerned in all developed countries and more stringent emissions regulations for motorcycles have been promulgated. THE CLOSED CHAMBER is a important test equipment for measuring fuel evaporative emissions of motorcycles and it should also be more sophisticated with strict regulations. Taking the Closed Chamber as object, this paper establishes the 3D model of the Closed Chamber and uses CFD simulation calculate flow distribution and gasoline vapor concentration distribution of the Closed Chamber and it provides a basis for solving practical engineering needs.

\section{SIMULATION ANALYSIS}

When applying CFD method to simulate the Flow distribution of the Closed Chamber, the basic equations and Theoretical equations are firstly established closed chamber. Starting from the basic principles (such as fluid mechanics, thermodynamics, heat and mass transfer and other conservation law), Conservation equations of turbulence characteristics method are built. These equations constitute a nonlinear partial differential equations, we cannot use the classical analytical method can only be solved by numerical methods. To solving the equations, the geometry and dimensions of the model must first be given, the calculated area and the proper import and export are determined. At the same time, the boundary conditions of the free surface are given. CFD simulation generally follows the following steps:

(1)Physical model of research subjects is first established closed chamber, and it becomes abstract to mathematical or mechanical model. Then its spatial influence area of model is determined.

(2) Establishing a CAD model of calculating area and gridding the outer surface of the grid geometry and the entire calculation area. Sparse grid and grid cell shape will have a huge impact on the future of computing. Different algorithms format to ensure the stability and efficiency calculations, the general requirements of the grid are not the same.

(3) Add Solution required initial conditions, such as boundary conditions at the inlet and the outlet is generally velocity pressure.

(4) Select the appropriate algorithm, some of the conditions set specific control procedures and the accuracy of solving the required analysis to solve problems and save the data file was found.

(5) Select the appropriate post-processor to read the results file, analysis and display.

\section{CAD MODEL}

Figures and tables, as originals of good quality and well contrasted, are to be in their final form, ready for reproduction, pasted in the appropriate place in the text. Try to ensure that the size of the text in your figures is approximately the same size as the main text (10 point). Try to ensure that lines are no thinner than 0.25 point.

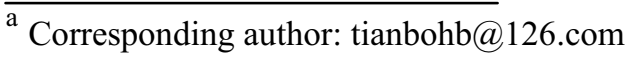




\subsection{Geometry}

Establish geometric model based on design drawings. Its size: $3000(\mathrm{~L}) \times 1800(\mathrm{~W}) \times 2000(\mathrm{H})$. Hexahedral and tetrahedral meshes mixed model mesh, the total number is about 850,000 grids.

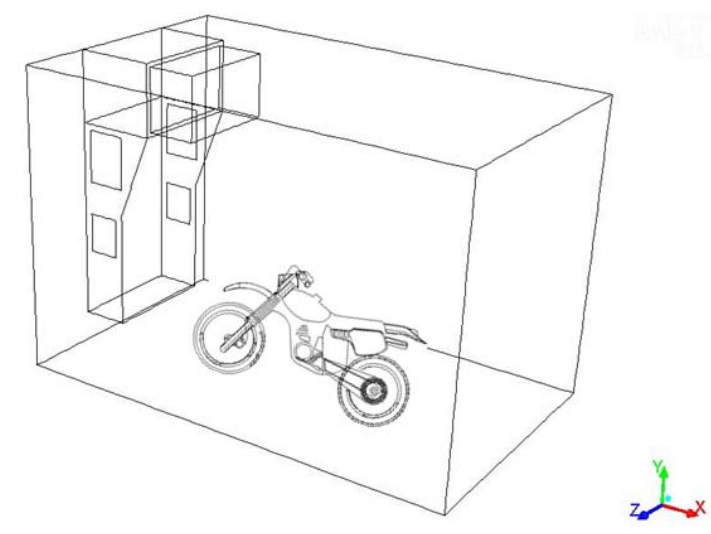

Figure 1. Geometry

\subsection{Mathematical Model}

According to the characteristics of the project, Selection the K-epsilon turbulence model:

$\frac{\partial}{\partial t}(\rho k)+\frac{\partial}{\partial x_{j}}\left(\rho k u_{i}\right)=\frac{\partial}{\partial x_{j}}\left[\left(\mu+\frac{\mu_{t}}{\sigma_{k}}\right) \frac{\partial k}{\partial x_{j}}\right]+G_{k}+G_{b}-\rho \varepsilon-Y_{H}+S_{k}$

$\frac{\partial}{\partial t}(\rho \varepsilon)+\frac{\partial}{\partial X_{i}}\left(\rho \varepsilon u_{i}\right)=\frac{\partial}{\partial X_{j}}\left[\left(\mu+\frac{\mu_{t}}{\sigma_{\varepsilon}}\right) \frac{\partial \varepsilon}{\partial X_{j}}\right]+G_{1 \varepsilon} \frac{\varepsilon}{k}\left(G_{k}+C_{3 \varepsilon} G_{b}\right)-C_{2 \varepsilon} \rho \frac{\varepsilon^{2}}{k}+S_{b}$

Where:

Gk: the turbulent kinetic energy represented by the laminar flow velocity gradient produced;

$\mathrm{Gb}$ : turbulent kinetic energy generated by buoyancy;

YM: compressible turbulence due to the fluctuation of the transition diffusion;

$\mathrm{C} 1, \mathrm{C} 2$ and $\mathrm{C} 3$ : the constant;

$\sigma \mathrm{k}, \sigma \mathrm{e}$ : turbulent Prandtl number of $\mathrm{k}$ equation and $\varepsilon$ equation;

Sk, Se: are for the user-defined.

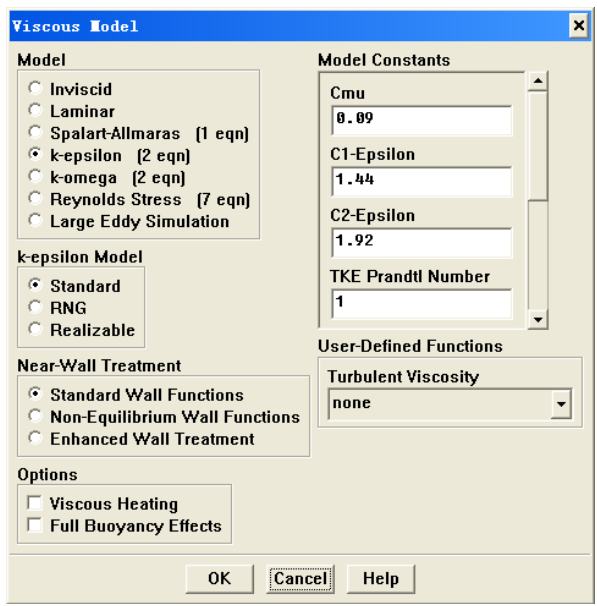

Figure 2. Mass transport model

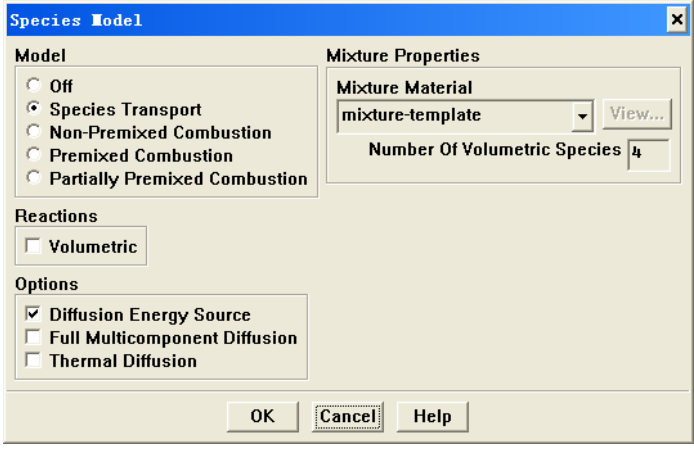

Figure 3. Gasoline vapor diffusion in air

\subsection{Boundary conditions}

Boundary conditions of the system are as follows:

i) Volatile border of gasoline vapor: Volatile gasoline vapor to the mass flow boundary conditions. The evaporation rate of gasoline is $2 \mathrm{~g} / \mathrm{h}$.

ii) Boundary fan: fan capacity: $2280 \mathrm{~m} 3 / \mathrm{h}$, wind speed: $2.87 \mathrm{~m} / \mathrm{s}$, wind pressure: $55 \mathrm{~Pa}$.

iii) Air Conditioning border: conditioned air volume: $2280 \mathrm{~m} 3$ / h, wind speed: $2.3 \mathrm{~m} / \mathrm{s}$, temperature: 15.5 $\sim 35.5$ degrees.

\section{RESULTS AND ANALYSIS}

To verify the reliability of the results of this simulation, the author conducted an actual experiment checked. Using $\mathrm{CH}$ analyzers to measuring concentration of $\mathrm{CH}$, and using handheld anemometer measuring wind speed. The boundary condition in the preamble is given by the measurement. All measuring points are taken as the height of the transverse plane $\mathrm{Y}=1.6 \mathrm{~m} \sim 1.8 \mathrm{~m}$, which the section according to $\mathrm{X}$ and $\mathrm{Z}$ directions equidistant take several measuring points respectively. During the experiment, the indoor flow distribution of $\mathrm{HC}$ is shown in Figure 4 and the concentration distribution of gasoline vapor is shown in Figure 5.

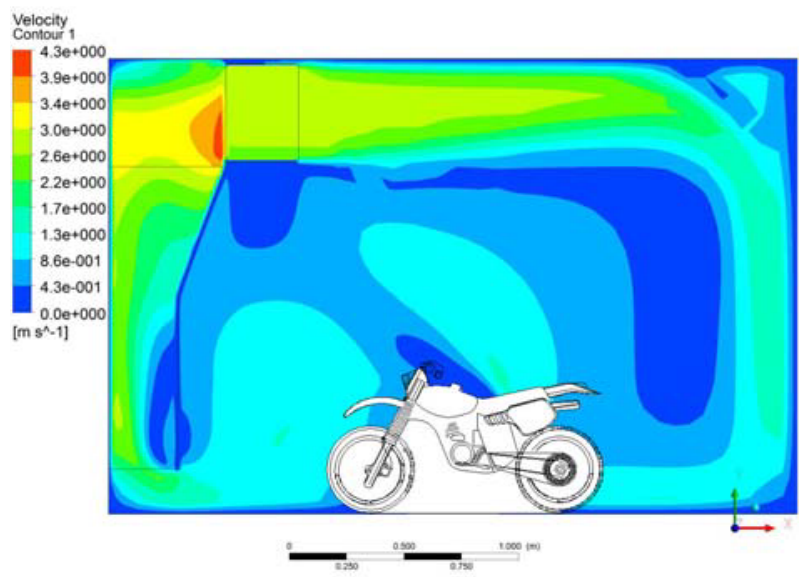

After 5 minutes 


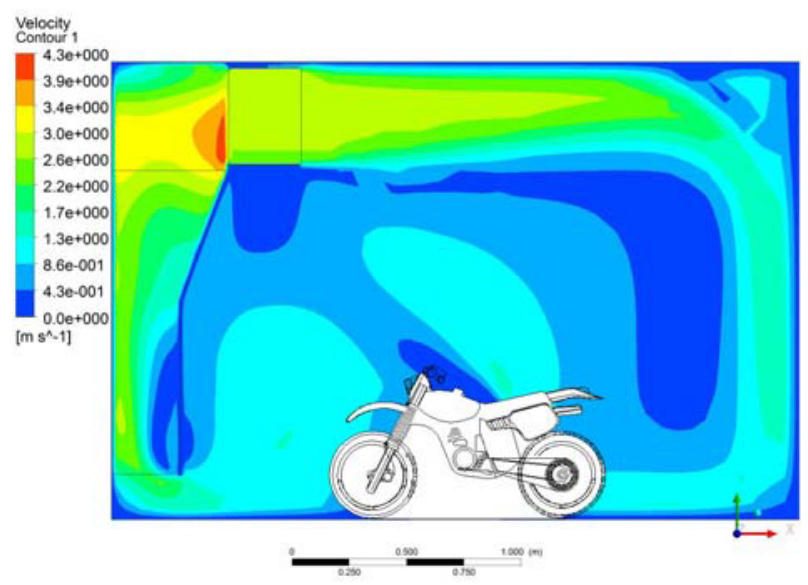

After 60 minutes

Figure 4. Flow distribution

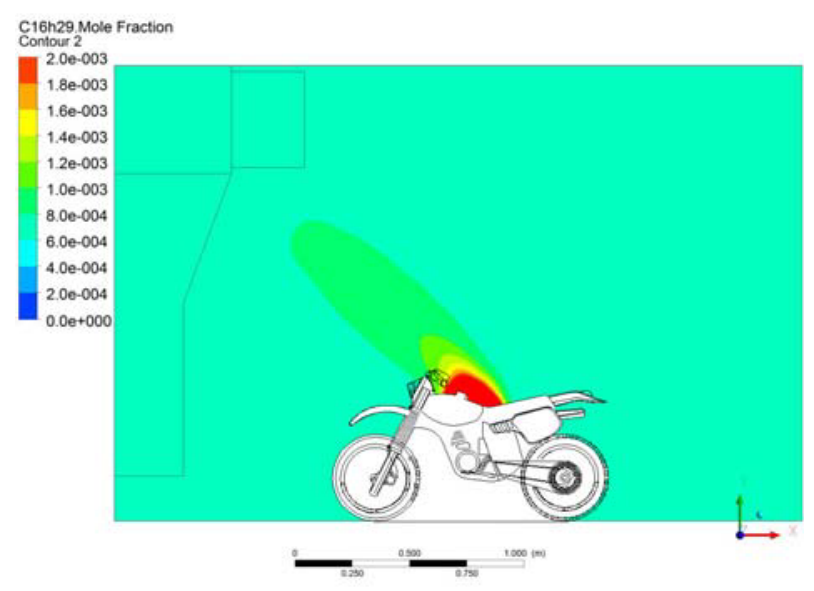

After 5 minutes

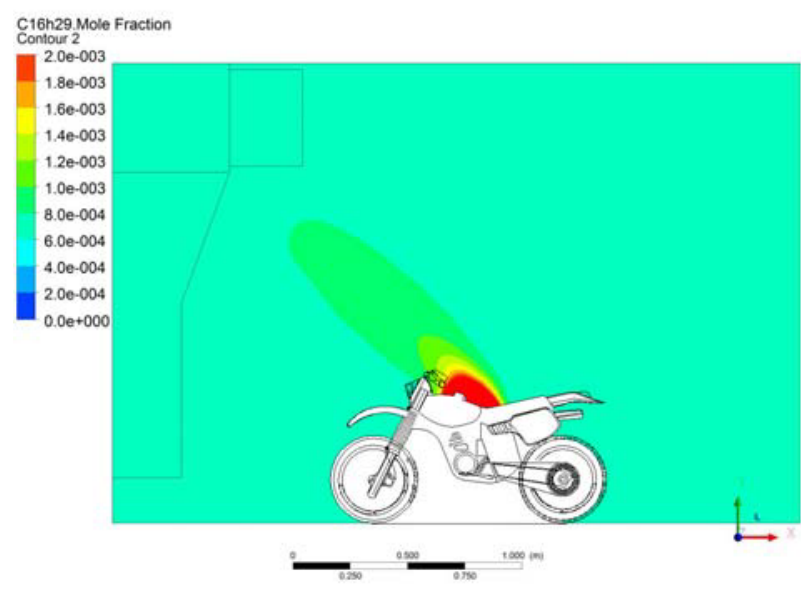

After 60 minutes

Figure 5. Gasoline Vapor Concentration Distribution

The above chart shows that the error of the edge indoor is larger. In addition to itself error of the testing equipment, the main reason is that the actual experimental control conditions is different from the simulation model. The simulation assumes that the flow is steady, that is, the speed and temperature values of each measuring point does not change with time. The interior is in fact due to the turbulent flow, velocity changes over time in the vicinity of the average random fluctuations. In addition, there is the time factor, as compared to temperature fluctuations over time smaller field, the error also small. The result is a simplified simulation model simulation and measured values. Another reason for the error, the problem will improve as well as improve the capacity of computer models of turbulence and continue to improve.

\section{CONCLUSION}

Taking the flow distribution as object, structural of the Closed Chamber improved to the containment chamber through the flow field analysis and it makes flow distribution more reasonable and significantly improves the uniformity of indoor gas concentration. The conclusions are summarized as follows: 1) With the conclusion of flow distribution and gasoline vapor concentration distribution of the Closed Chamber reached via the CFD software of Fluent and experiment research, this paper analyzes the flow distribution and concentration distribution of gasoline vapor. 2) CFD simulation showed that: the rational design of the Closed Chamber is the key factor to ensure a uniform concentration of indoor gasoline. Reasonable flow rate can significantly improve the indoor gas concentration uniformity. The practical application of these simulation methods improved the performance and reliability of the Closed Chamber.

\section{References}

1. SUN Hailong. The CFD Analysis Computation and Optimization Research of Locomotive Electrical Cabinet Ventilation System[D]. Dalian Jiaotong University, 2015.

2. Tan Linbo, Liu Shengji, Lu Cunhao, Wang Jian. CFD analysis and experiment research of non-road gasoline engine cooling system[J]. Journal of Chinese Agricultural Mechanization, 2014, 35(1): 189-192.

3. Miltner M, Jordan C, Harasek M. CFD Simulation of straight and slightly swirling turbulent free jets using different RANS-Turbulence models[J]. Applied Thermal Engineering, 2015, 89:1117-1126.

4. Vyskocil L, Schmid J, Macek J. CFD simulation of air-steam flow with condensation[J]. Nuclear Engineering \& Design, 2014, 279:147-157.

5. Nesiadis Athanasios, Nikolopoulos Nikolaos, Margaritis Nikolaos, et al. Optimization of a log wood boiler through CFD simulation methods[J]. Fuel Processing Technology, 2015, 137:75-92.

6. Jia Quanli. A brief introduction to the test method for fuel evaporative emission of motorcycle(II)[J]. Motorcycle Technology, 2009, 11:38-41.

7. Qiu Jie. Quality of fuel evaporative controlling system of motorcycle and study on related problem[J]. Motorcycle Technology, 2007, 5:16-19.

8. Terashima M, Goel R, Komatsu K, et al. CFD simulation of mixing in anaerobic digesters[J]. Bioresource Technology, 2009, 100(7):2228-2233. 